\title{
The relationships between parenting styles and motivation orientations, body mass index and nutrition behaviour of UK university students
}

\author{
M. Pendlebury ${ }^{1}$, N. Lowe ${ }^{2}$, E. Westaway ${ }^{2}$ and F. Amirabdollahian ${ }^{1}$ \\ ${ }^{1}$ School of Health Sciences, Liverpool Hope University, Liverpool L16 $9 \mathrm{JD}$ and ${ }^{2}$ International Institute of Nutritional \\ Sciences and Applied Food Safety Studies, University of Central Lancashire, Preston, PR1 2HE, UK
}

The motivational elements of the Self Determination Theory ${ }^{(1)}$ proposes that early exposures to overly controlling, and non-autonomy supportive parenting styles are maladaptive to offspring's intrinsic motivation and self-regulation ${ }^{(2)}$. These have also been previously linked to unhealthy nutrition behaviours and risk of becoming overweight ${ }^{(3)}$. When reaching emerging adulthood (ages 18-25), and attending university, offspring's parental control often reduces, whilst challenges for behavioural self-regulation increases ${ }^{(4)}$. It was previously demonstrated that university students tend to consume excessive amounts of convenience foods and alcohol ${ }^{(5)}$; however, evidence on relationships between parental autonomy support (AS) and psychological control (PC) in childhood with nutrition behaviour in young adulthood is scarce and inconclusive ${ }^{(6)}$. The aim of the present study was to investigate how young students' experiences of parental AS and PC were related to their present intrinsic motivations (IM), unhealthy nutrition behaviours, and Body Mass Index (BMI).

Participants $(n=71)$ from two universities, completed two validated parenting style questionnaires as well as a comprehensive lifestyle survey including present intrinsic motivations. Weight and height were measured and intakes of alcohol, salt, energy, and contribution of saturated fat (SF) and non-milk extrinsic sugars (NMES) to overall energy intake were estimated using a 3-day dietary record. Kolmogorov-Smirnov test was used for assessment of the normal distributions and Pearson's correlations coefficient was used for exploration of the relationships.

Weak to moderate but significant inverse relationships were recorded between the current IM and BMI, daily intake of salt and percentage contribution of saturated fat to overall energy intake. Parenting style (especially maternal PC and AS) was found to be significantly associated with IM and this association was positive between IM and AS, and negative between IM and PC. Maternal PC and AS were also moderately associated with percentage contribution of SF to overall energy intake and weakly associated with salt intake. No significant associations were found between IM or parenting styles, neither with the consumption of alcohol nor the contribution of NMES to overall energy intake.

\begin{tabular}{|c|c|c|c|c|c|c|}
\hline & \multicolumn{6}{|c|}{ Association (Pearson Correlation Coefficient) *significant at 5\%, **significant at $1 \%, * * *$ significant at $0.1 \%$} \\
\hline & Present IM score & BMI $\left(\mathrm{kg} / \mathrm{m}^{2}\right)$ & Energy from SF \% & Energy from NMES \% & Salt $(\mathrm{g} / \mathrm{d})$ & Alcohol (unit/session) \\
\hline Present IM score & - & $-0.32 *$ & $-0.50 * * *$ & -0.28 & $-0.27 *$ & -0.21 \\
\hline \multicolumn{7}{|l|}{ Parenting style } \\
\hline Maternal PC score & $-0.66^{* * *}$ & $0.34 *$ & $0.55 * * *$ & 0.04 & $0.36^{* *}$ & 0.17 \\
\hline Maternal AS score & $0.58 * * *$ & $0.26^{*}$ & $-0.51 * * *$ & -0.01 & $-0.24 *$ & -0.20 \\
\hline Paternal PC score & $-0.38^{*}$ & $0.24 *$ & $0.32 *$ & -0.01 & $0.30 *$ & 0.22 \\
\hline Paternal AS score & $0.30 *$ & 0.15 & 0.26 & 0.22 & -0.15 & -0.06 \\
\hline
\end{tabular}

The current findings suggest that parental PC and AS may have a role in aetiology of unhealthy nutrition behaviour in emerging adulthood and the influence of these might be through the medium of the IM. It may therefore be of benefit to reduce young children's exposures to autonomy obstructing and psychologically controlling parenting styles to enhance IM and self-regulation in later life.

1. Deci EL \& Ryan RM (2000) Psychol Inq 11, 227-268.

2. Silk JS, Morris AS, Kanaya T et al. (2003) J Res Adolesc 1, 113-128.

3. Ventura AK \& Birch LL (2008) Int J Behav Nutr Phys Act 5, 15.

4. Arnett JJ (2000) Am Psychol 5, 469-480.

5. Hoffman DJ, Policastro P, Quick V et al. (2006) J Am Coll Health 55, 41-44.

6. McSweeney LA, Stamp E, Jones AR et al. (2010) Proc Nutr Soc 69, E417. 